\title{
A Pressure-Correction Scheme for Rotational Navier-Stokes Equations and Its Application to Rotating Turbulent Flows
}

\author{
Dinesh A. Shetty ${ }^{1}$, Jie Shen ${ }^{2,3, *}$, Abhilash J. Chandy ${ }^{4}$ and \\ Steven H. Frankel ${ }^{1}$ \\ ${ }^{1}$ School of Mechanical Engineering, Purdue University, West Lafayette, \\ IN 47907, USA. \\ ${ }^{2}$ School of Mathematical Sciences, Xiamen University, Xiamen, China. \\ ${ }^{3}$ Department of Mathematics, Purdue University, West Lafayette, IN 47907, USA. \\ ${ }^{4}$ Department of Mechanical Engineering, University of Akron, Akron, \\ $\mathrm{OH} 44325$, USA.
}

Received 30 November 2009; Accepted (in revised version) 4 March 2010

Available online 17 September 2010

To the memory of David Gottlieb

\begin{abstract}
The rotational incremental pressure-correction (RIPC) scheme, described in Timmermans et al. [Int. J. Numer. Methods. Fluids., 22 (1996)] and Shen et al. [Math. Comput., 73 (2003)] for non-rotational Navier-Stokes equations, is extended to rotating incompressible flows. The method is implemented in the context of a pseudo Fourier-spectral code and applied to several rotating laminar and turbulent flows. The performance of the scheme and the computational results are compared to the socalled diagonalization method (DM) developed by Morinishi et al. [Int. J. Heat. Fluid. Flow., 22 (2001)]. The RIPC predictions are in excellent agreement with the DM predictions, while being simpler to implement and computationally more efficient. The RIPC scheme is not in anyway limited to implementation in a pseudo-spectral code or periodic boundary conditions, and can be used in complex geometries and with other suitable boundary conditions.
\end{abstract}

AMS subject classifications: 52B10, 65D18, 68U05, 68U07

Key words: Rotating turbulent flow, pressure-correction, Navier-Stokes equations, Fourier-spectral method.

\section{Introduction}

The ability to accurately and efficiently incorporate the effects of rotation in numerical simulations of fluid flows is important in a number of different science and engineering

${ }^{*}$ Corresponding author. Email addresses: dshetty@purdue.edu (D. A. Shetty), shen7@purdue.edu (J. Shen), ac76@uakron.edu (A. J. Chandy), frankel@ecn.purdue.edu (S. H. Frankel) 
applications including atmospheric, oceanic, astrophysical, internal combustion engine, and turbo-machinery flows. Fundamental studies of rotating turbulence are also important for improved understanding and modeling of such flows [1]. The numerical challenge is related to the presence of the Coriolis term in the Navier-Stokes equations. Explicit treatment of this term in time limits simulations to low rotation rates and/or small time steps. This suggests that an implicit treatment of the Coriolis term is essential for stable, affordable, and realistic simulations of rotating flows. For the case of rotating homogeneous turbulence, Diagonalization Methods (DM) are often used to perform exact integration of the rotation terms [2-4]. These methods analytically integrate the Coriolis terms to provide an accurate solution. However, due to its special form, DM methods can only be applied to problems with periodic boundary conditions and is somewhat cumbersome to implement. Also, the DM method can not be easily extended to other type of boundary conditions and/or complex geometries.

In the present study, we develop a rotational incremental pressure correction (RIPC) method which treats the rotation term implicitly while being accurate and computationally efficient, and show the mathematical analysis of stability of the scheme. To avoid confusion, it is worth mentioning here that the word rotational in the name of the scheme refers to the use of the rotational form of the diffusion operator in the incompressible Navier-Stokes equations, whereas the word rotation refers to solid-body rotation of the flow itself. Predictions from the RIPC scheme are compared to the DM method of Morinishi et al. [4] with regard to accuracy versus efficiency. All computations in this study are performed in the context of a Fourier pseudo-spectral simulation but are in no way limited to this numerical discretization scheme or to the use of periodic boundary conditions.

The rest of the paper is organized as follows. In the next section, we introduce the RIPC scheme with rotation and prove its stability. In Section 3, we validate the accuracy and stability of the scheme against the DM scheme in [4]. We then present some direct numerical simulation (DNS) and large eddy simulation (LES) results for rotating turbulent flows in Section 4. We conclude with a few remarks in Section 5.

\section{Mathematical formulation}

\subsection{Governing equations}

The incompressible Navier-Stokes equations with rotation are given by the following:

$$
\begin{aligned}
& \frac{\partial u_{i}}{\partial x_{i}}=0, \\
& \frac{\partial u_{i}}{\partial t}+u_{j} \frac{\partial u_{i}}{\partial x_{j}}=-\frac{\partial p}{\partial x_{i}}+v \frac{\partial^{2} u_{i}}{\partial x_{j} \partial x_{j}}-\left(\boldsymbol{\Omega}_{\mathbf{j}} \times \mathbf{u}\right)_{i},
\end{aligned}
$$

where $u_{i}(i=1,2,3)$ are the components of the velocity field $\mathbf{u}, p$ is the effective pressure, $v$ is the kinematic viscosity, $\Omega=\left(\Omega_{1}, \Omega_{2}, \Omega_{3}\right)$ is the rotation vector. Here we only study the 
case where $\Omega=(0,0, \Omega)$. Hence, the Coriolis term on the right hand side of the NavierStokes equations simplifies to $-2 \Omega \epsilon_{i 3 k} u_{k}$. Eq. (2.1b) should be supplemented with a set of suitable boundary conditions for the velocity field.

\subsection{RIPC method}

One of the major difficulties with numerical simulation of incompressible flows is the coupled nature of the velocity and pressure fields due to the incompressibility constraint. A popular and effective way to decouple the computation of the pressure from that of the velocity is to use a projection type scheme originally proposed by Chorin [5] and Temam [6]. An overview of projection schemes for incompressible flows can be found in [7] where the projection schemes are classified into three classes: pressure-correction, velocity-correction and consistent splitting, each with standard and rotational formulation. In the current work, the RIPC scheme proposed by Timmermans et al. [8] (see also [9]) is extended for rotating flows. A detailed description of the RIPC scheme for non-rotating flows and an error analysis can be found in [9].

The RIPC scheme with a Backward Difference Formula (BDF) for Eq. (2.1b) (with $\mathbf{U}=[u, v, w]^{T}$ and $\tau$ being time step) is shown below:

1. In the first substep, we find $\tilde{\mathbf{U}}=\left(\tilde{u}^{n+1}, \tilde{v}^{n+1}, \tilde{w}^{n+1}\right)$ by solving:

$$
\begin{aligned}
& \frac{a_{1} \tilde{u}^{n+1}-\sum_{i=2}^{q+1} a_{i} u^{n+2-i}}{\tau}-v \Delta \tilde{u}^{n+1}-2 \Omega \tilde{v}^{n+1}+\frac{\partial p^{\star, n+1}}{\partial x}+\mathrm{NLT}_{1}=0, \\
& \frac{a_{1} \tilde{v}^{n+1}-\sum_{i=2}^{q+1} a_{i} v^{n+2-i}}{\tau}-v \Delta \tilde{v}^{n+1}+2 \Omega \tilde{u}^{n+1}+\frac{\partial p^{\star, n+1}}{\partial y}+\mathrm{NLT}_{2}=0 \\
& \frac{a_{1} \tilde{w}^{n+1}-\sum_{i=2}^{q+1} a_{i} w^{n+2-i}}{\tau}-v \Delta \tilde{w}^{n+1}+\frac{\partial p^{\star, n+1}}{\partial z}+\mathrm{NLT}_{3}=0 .
\end{aligned}
$$

In the above, $\left(\tilde{u}^{n+1}, \tilde{v}^{n+1}, \tilde{w}^{n+1}\right)$ satisfy the boundary condition of $\mathbf{U}\left(\cdot, t^{n+1}\right)$.

2. In the second substep, we find $\left(\mathbf{U}^{n+1}, \phi^{n+1}\right)$ from

$$
\begin{aligned}
& \frac{a_{1}\left(\mathbf{U}^{n+1}-\tilde{\mathbf{U}}^{n+1}\right)}{\tau}+\nabla \phi^{n+1}=0, \\
& \nabla \cdot \mathbf{U}^{n+1}=0 .
\end{aligned}
$$

The correct boundary condition for $(2.3 a)-(2.3 b)$ is that the normal component of $U^{n+1}$ equals to the normal component of $U\left(\cdot, t^{n+1}\right)$ at the boundary.

3. In the final substep, we update $p^{n+1}$ by

$$
p^{n+1}=\phi^{n+1}+p^{\star, n+1}-v \nabla \cdot \tilde{\mathbf{U}}^{n+1} .
$$


In the above scheme, $\left\{a_{i}\right\}_{i=1}^{q+1}$ are chosen to form the $q$-th order BDF, namely,

$$
\begin{array}{lll}
q=2: & a_{1}=\frac{3}{2}, \quad a_{2}=2, \quad a_{3}=-\frac{1}{2}, \\
q=3: & a_{1}=\frac{11}{6}, \quad a_{2}=3, \quad a_{3}=-\frac{3}{2}, \quad a_{4}=\frac{1}{3} ;
\end{array}
$$

$p^{\star, n+1}$ is the $r^{\text {th }}$-order extrapolation for $p^{n+1}$ :

$$
p^{\star, n+1}= \begin{cases}p^{n}, & \text { if } r=1, \\ 2 p^{n}-p^{n-1}, & \text { if } r=2 ;\end{cases}
$$

and $\mathrm{NLT}_{i}$ are the $q$-th order extrapolated nonlinear terms, namely,

$$
\begin{aligned}
& q=2: \mathrm{NLT}_{1}=2\left(\mathbf{U}^{\mathbf{n}} \cdot \nabla\right) \tilde{u}^{n}-\left(\mathbf{U}^{n-1} \cdot \nabla\right) \tilde{u}^{n-1}, \\
& q=3: \mathrm{NLT}_{1}=3\left(\mathbf{U}^{\mathbf{n}} \cdot \nabla\right) \tilde{u}^{n}-3\left(\mathbf{U}^{n-1} \cdot \nabla\right) \tilde{u}^{n-1}+\left(\mathbf{U}^{n-2} \cdot \nabla\right) \tilde{u}^{n-2},
\end{aligned}
$$

similarly for $\mathrm{NLT}_{2}$ and $\mathrm{NLT}_{3}$.

Several comments are in order:

- In general, taking $r=q-1$ will lead to a $q$-th order scheme for the velocity (in the $L^{2}$-norm) but not necessarily for the pressure; but taking $r=q$ will lead to a formally $q$-th order scheme for both the velocity and pressure. We refer to [7] for more detail.

- In the above scheme, the rotational and viscous terms are treated implicitly, thus removing the time step limitation associated with these two terms in the Navier-Stokes equations.

- Eqs. (2.2a) and (2.2b) are coupled by the rotation. But since the coupled system is elliptic, their simultaneous solution can be easily accomplished by using a standard finite element method or a spectral method. In particular, we provide a fast Fourier-spectral algorithm at the end of this section for the case of periodic boundary conditions. Effective spectral algorithms can also be constructed for other type of boundary conditions, such as mixed periodic-Dirichlet boundary conditions for channel flows or no-slip Dirichlet boundary conditions, by using the spectral-Galerkin methods [10,11].

- The system (2.3a)-(2.3b) can be easily decoupled. In fact, using the divergence free condition, a Poisson equation can be obtained for $\phi^{n+1}$ :

$$
\nabla^{2} \phi^{n+1}=\frac{a_{1}}{\tau} \nabla \cdot \tilde{\mathbf{U}}^{n+1},
$$

with homogeneous Neumann boundary conditions for $\phi^{n+1}$.

\subsection{Stability proof}

We will sketch, in this subsection, a proof for the stability of the RIPC scheme applied to the forced Stokes equations in a simple setting, namely, the case with $(q, r)=(1,1)$. 
To fix the idea, we shall assume that the velocity field satisfies the homogeneous no-slip boundary condition. We note that the proof below, inspired by the proof in [9] for the RIPC scheme for the non-rotational Navier-Stokes equations, carries over to the case of periodic boundary conditions without modification.

To simplify the presentation, we rewrite the scheme with $(q, r)=(1,1)$ for the forced Stokes equations in the following vector form:

$$
\begin{aligned}
& \left\{\begin{array}{l}
\frac{\tilde{u}^{k+1}-u^{k}}{\tau}-v \Delta \tilde{u}^{k+1}+\boldsymbol{\Omega} \times \tilde{u}^{k+1}+\nabla p^{k}=f\left(t_{k+1}\right), \\
\left.\tilde{u}^{k+1}\right|_{\Gamma}=0,
\end{array}\right. \\
& \left\{\begin{array}{l}
\frac{u^{k+1}-\tilde{u}^{k+1}}{\tau}+\nabla\left(p^{k+1}-p^{k}+v \nabla \cdot \tilde{u}^{k+1}\right)=0, \\
\nabla \cdot u^{k+1}=0, \\
\left.u^{k+1} \cdot n\right|_{\Gamma}=0 .
\end{array}\right.
\end{aligned}
$$

Let us introduce some notations. We denote

$$
(u, v)=\int_{D} u v d x
$$

where $D \subset \mathbb{R}^{d}$ is the region occupied by the fluid, and $\|u\|^{2}=(u, v)$. For any sequence of functions $\phi^{k}$, we denote

$$
\delta_{t} \phi^{k}=\phi^{k}-\phi^{k-1}
$$

Applying the operator $\delta_{t}$ to (2.9a), we find

$$
\left\{\begin{array}{l}
\frac{\delta_{t} \tilde{u}^{k+1}-\delta_{t} u^{k}}{\tau}-v \Delta \delta_{t} \tilde{u}^{k+1}+\boldsymbol{\Omega} \times \delta_{t} \tilde{u}^{k+1}+\delta_{t} \nabla p^{k}=\delta_{t} f\left(t_{k+1}\right), \\
\left.\delta_{t} \tilde{u}^{k+1}\right|_{\Gamma}=0
\end{array}\right.
$$

Next, we apply the operator $\delta_{t}$ to $(2.9 \mathrm{~b})$ and rearrange the resultant system to:

$$
\left\{\begin{array}{l}
\frac{\delta_{t} u^{k+1}}{\tau}+\nabla\left(\delta_{t} p^{k+1}+v \nabla \cdot \tilde{u}^{k+1}\right)=\frac{\delta_{t} \tilde{u}^{k+1}}{\tau}+\nabla\left(\delta_{t} p^{k}+v \nabla \cdot \tilde{u}^{k}\right), \\
\nabla \cdot u^{k+1}=0 \\
\left.u^{k+1} \cdot n\right|_{\Gamma}=0 .
\end{array}\right.
$$

Taking the inner-product of (2.10) with $2 \tau \delta_{t} \tilde{u}^{k+1}$, and using the fact that

$$
(\boldsymbol{\Omega} \times u, u)=0, \quad \forall u \in L^{2}(D)^{d},
$$

we find

$$
\begin{gathered}
\left\|\delta_{t} \tilde{u}^{k+1}\right\|^{2}-\left\|\delta_{t} u^{k}\right\|^{2}+\left\|\delta_{t} \tilde{u}^{k+1}-\delta_{t} u^{k}\right\|^{2}+2 v \tau\left\|\nabla \delta_{t} \tilde{u}^{k+1}\right\|^{2} \\
+2 \tau\left(\nabla \delta_{t} p^{k}, \delta_{t} \tilde{u}^{k+1}\right)=2 \tau\left(\delta_{t} f^{k+1}, \delta_{t} \tilde{u}^{k+1}\right) .
\end{gathered}
$$


Taking the inner product of each side of (2.11) with itself, and using the fact that $\nabla \cdot u^{k+1}=$ 0 , we find

$$
\begin{aligned}
& \left\|\delta_{t} u^{k+1}\right\|^{2}+\tau^{2}\left\|\nabla\left(\delta_{t} p^{k+1}+v \nabla \cdot \tilde{u}^{k+1}\right)\right\|^{2} \\
= & \left\|\delta_{t} \tilde{u}^{k+1}\right\|^{2}+\tau^{2}\left\|\nabla\left(\delta_{t} p^{k}+v \nabla \cdot \tilde{u}^{k}\right)\right\|^{2}+2 \tau\left(\nabla\left(\delta_{t} p^{k}+v \nabla \cdot \tilde{u}^{k}\right), \delta_{t} \tilde{u}^{k+1}\right) .
\end{aligned}
$$

Summing up the last two equations, we arrive at

$$
\begin{aligned}
& \left\|\delta_{t} u^{k+1}\right\|^{2}-\left\|\delta_{t} u^{k}\right\|^{2}+\left\|\delta_{t} \tilde{u}^{k+1}-\delta_{t} u^{k}\right\|^{2}+2 v \tau\left\|\nabla \delta_{t} \tilde{u}^{k+1}\right\|^{2} \\
& \quad+\tau^{2}\left(\left\|\nabla\left(\delta_{t} p^{k+1}+v \nabla \cdot \tilde{u}^{k+1}\right)\right\|^{2}-\left\|\nabla\left(\delta_{t} p^{k}+v \nabla \cdot \tilde{u}^{k}\right)\right\|^{2}\right) \\
& =2 \tau\left(\delta_{t} \tilde{u}^{k+1}, \nabla \nabla \cdot u^{k}\right)+2 \tau\left(\delta_{t} f^{k+1}, \delta_{t} \tilde{u}^{k+1}\right) .
\end{aligned}
$$

Integrating by parts in the first term on the right hand side, we find

$$
\begin{aligned}
2 \tau\left(\delta_{t} \tilde{u}^{k+1}, \nabla \nabla \cdot u^{k}\right) & =-2 \Delta t\left(\nabla \cdot \delta_{t} \tilde{u}^{k+1}, \nabla \cdot u^{k}\right) \\
& =-\tau\left(\left\|\nabla \cdot \tilde{u}^{k+1}\right\|^{2}-\left\|\nabla \cdot \tilde{u}^{k}\right\|^{2}-\left\|\nabla \cdot \delta_{t} \tilde{u}^{k+1}\right\|^{2}\right) .
\end{aligned}
$$

Plugging in the above in (2.14), and using the identity (cf. [12])

$$
\|\nabla u\|^{2}=\|\nabla \times u\|^{2}+\|\nabla \cdot u\|^{2}, \quad \forall u \in H_{0}^{1}(D)^{d},
$$

we derive

$$
\begin{aligned}
& \quad\left\|\delta_{t} u^{k+1}\right\|^{2}-\left\|\delta_{t} u^{k}\right\|^{2}+\left\|\delta_{t} \tilde{u}^{k+1}-\delta_{t} u^{k}\right\|^{2}+v \tau\left\|\nabla \delta_{t} \tilde{u}^{k+1}\right\|^{2} \\
& \quad+v \tau\left\|\nabla \times \delta_{t} \tilde{u}^{k+1}\right\|^{2}+\tau^{2}\left(\left\|\nabla\left(\delta_{t} p^{k+1}+v \nabla \cdot \tilde{u}^{k+1}\right)\right\|^{2}-\left\|\nabla\left(\delta_{t} p^{k}+v \nabla \cdot \tilde{u}^{k}\right)\right\|^{2}\right) \\
& =2 \tau\left(\delta_{t} f^{k+1}, \delta_{t} \tilde{u}^{k+1}\right) \leq \frac{C}{v} \tau\left\|\delta_{t} f^{k+1}\right\|^{2}+\frac{v \tau}{2}\left\|\nabla \delta_{t} \tilde{u}^{k+1}\right\|^{2},
\end{aligned}
$$

where $C=2 C_{1}$ with $C_{1}$ being the constant in the Poincare inequality (cf. [12]).

Summing up the above relation for $k=1,2, \cdots, n$, we have proved the following stability result:

$$
\begin{aligned}
& \left\|\delta_{t} u^{n+1}\right\|^{2}+\tau^{2}\left\|\nabla\left(\delta_{t} p^{n+1}+v \nabla \cdot \tilde{u}^{n+1}\right)\right\|^{2}+v \tau \sum_{k=1}^{n}\left\|\nabla \delta_{t} \tilde{u}^{k+1}\right\|^{2}+\sum_{k=1}^{n}\left\|\delta_{t} \tilde{u}^{k+1}-\delta_{t} u^{k}\right\|^{2} \\
\leq & \left\|\delta_{t} u^{1}\right\|^{2}+\tau^{2}\left\|\nabla\left(\delta_{t} p^{1}+v \nabla \cdot \tilde{u}^{1}\right)\right\|^{2}+\frac{C \tau}{v} \sum_{k=1}^{n}\left\|\delta_{t} f^{k+1}\right\|^{2}, \quad \forall n \geq 1 .
\end{aligned}
$$

Several remarks are in order:

- The above inequality indicates that the scheme (2.9a)-(2.9b) is unconditionally stable, despite the fact that the pressure is treated explicitly in (2.9a).

- Due to the explicit treatment of the nonlinear terms, the RIPC scheme for the nonlinear Navier-Stokes equations is subjected to the usual CFL stability condition.

- By using essentially the same argument in the above and in [9], albeit with more involved calculations, we can also prove that the RIPC scheme with $(q, r)=(2,1)$ for the forced Stokes equations is unconditionally stable. 


\subsection{A fast pseudo Fourier-spectral algorithm in the periodic case}

Since we are interested in the simulation of homogeneous rotating turbulence, we now present implementation of the RIPC scheme with periodic boundary conditions in the context of a Fourier pseudo-spectral code. The algorithm below is presented in Fourier wavenumber space.

Assuming a constant rotating rate $\Omega$, for any wave number $k$, the $k$-th component of the Fourier transform of (2.2a) becomes

$$
\left(\frac{a_{1}}{\tau}+v k^{2}\right) \hat{\tilde{u}}_{k}^{n+1}+b_{1} \hat{\vec{v}}_{k}^{n+1}=\mathcal{F}_{k}\left\{-\frac{\partial p^{\star, n+1}}{\partial x}-\mathrm{NLT}_{1}+\frac{\sum_{i=2}^{q+1} a_{i} u^{n+2-i}}{\tau}\right\}
$$

where $\hat{u}_{k}, \hat{v}_{k}$ represent the $k$-th Fourier coefficients of the $\tilde{u}^{n+1}$ and $\tilde{v}^{n+1}$, respectively, and $\mathcal{F}_{k}$ is the $k$-th component of the Fourier transform. Similar equations for the other velocity components can be written.

Defining

$$
c=\frac{a_{1}}{\tau}+v k^{2}, \quad r(\hat{u})_{k}=\mathcal{F}_{k}\left\{-\frac{\partial p^{\star, n+1}}{\partial x}-\mathrm{NLT}_{1}+\frac{\sum_{i=2}^{q+1} a_{i} u^{n+2-i}}{\tau}\right\},
$$

and similarly for $r(\hat{v})_{k}, r(\hat{w})_{k}$, we find that (2.2a), (2.2b) and (2.2c) reduces to a sequence (with index $k$ ) of systems:

$$
\left[\begin{array}{ccc}
c & -2 \Omega & 0 \\
2 \Omega & c & 0 \\
0 & 0 & c
\end{array}\right]\left[\begin{array}{c}
\tilde{u}_{k}^{n+1} \\
\tilde{v}_{k}^{n+1} \\
\tilde{w}_{k}^{n+1}
\end{array}\right]=\left[\begin{array}{c}
r(\hat{u})_{k} \\
r(\hat{v})_{k} \\
r(\hat{w})_{k}
\end{array}\right]
$$

It is clear that the above system can be easily solved for each $k$.

We note that in the non-periodic case, the coupled linear system in (2.2) can not be solved as easily as above in the periodic case. However, at least for regular domains, this coupled system can still be solved efficiently by using a new spectral method at a cost comparable to that of a decoupled system (cf. [13]).

\section{Validation}

An in-house Fourier pseudo-spectral code numerically integrates the incompressible Navier-Stokes equations in a $(0,2 \pi)^{3}$ periodic box is the computational platform used for validation purposes. The non-linear convective terms are computed in physical space and the $2 / 3$ rule is used for aliasing error removal [14]. The code was recently validated in the context of large eddy simulations of homogeneous turbulence using both experimental measurements in grid-generated turbulence and direct numerical simulation data. Further details about the code and the validation studies can be found in [15]. 
The DM algorithm, proposed by Morinishi et al. [4], is also implemented here to serve as a benchmark for comparison. In DM, the Navier-Stokes equations are first diagonalized through a transformation technique described in [4]. The transformed equations are then integrated using an integration factor approach, which facilitates analytic integration of both the viscous and Coriolis terms, hence removing the associated time-step limitation. Note that the DM scheme can not be extended to other type of boundary conditions.

In all simulations, a third-order Runge-Kutta scheme [16] is used for time advancement in the DM scheme, while different sets of $(q, r)$ are used for the RIPC scheme. More precisely, to compare the accuracy of DM and RIPC schemes for the forced Stokes problems, we use $(q, r)=(3,2)$ and $(3,1)$ which are formally third-order and second-order, respectively. However, our numerical experiments indicate using the third-order extrapolations for the pressure and nonlinear terms may lead to instabilities. So for nonlinear simulations presented below, we use $(q, r)=(2,2)$ and $(2,1)$ which are both second-order but the former is more accurate with smaller truncation errors, but may require a smaller time step to be stable.

We shall use three discrete vector norms, namely $L_{\infty}, L_{2}$ and $H^{1}$, evaluated at the Fourier-collocation points, to assess the efficiency of the schemes.

\subsection{Comparison with an exact solution of the Stokes problem}

In this subsection, we compare the accuracy of our RIPC scheme with the DM scheme.

The first case in this section involves the forced Stokes equations:

$$
\begin{aligned}
& \frac{\partial u_{i}}{\partial x_{i}}=0, \\
& \frac{\partial u_{i}}{\partial t}=-\frac{\partial p}{\partial x_{i}}+v \frac{\partial^{2} u_{i}}{\partial x_{j} \partial x_{j}}-2 \epsilon_{i j k} \Omega_{j} u_{k}+f_{i},
\end{aligned}
$$

where $\Omega=(0,0, \Omega)$ and the forcing functions are such that the solution to (3.1a)-(3.1b) is:

$$
\begin{aligned}
& u=\sin (x) \cos (y) \cos (z) e^{\sin (t)}, \\
& v=-\cos (x) \sin (y) \cos (z) e^{\sin (t)}, \\
& w=0, \\
& p=\sin (x) \cos (y) \sin (z) e^{\sin (t)} .
\end{aligned}
$$

Simulations are performed with 120 grid points in each of the three coordinate directions and a constant time-step, $\tau$. The $L_{2}$ error norms the computed to the exact solution are reported in Fig. 1. We notice that the RIPC scheme with $(q, r)=(3,2)$ is as accurate as the DM scheme. In order to illustrate the influence of the value $r$, we also plotted the results of RIPC with $(q, r)=(3,1)$. It is observed that the convergence rate of RIPC scheme is, as expected, of order $\min (q, r+1)$. 


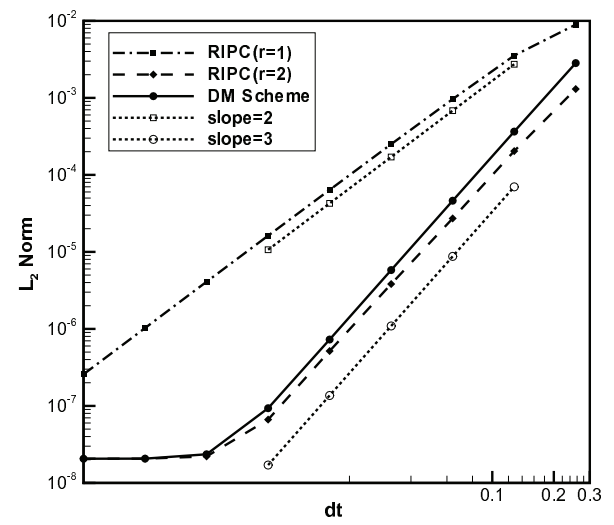

Figure 1: $L_{2}$ Error Norm plot.

In the next set of simulations, we vary the rotation rate $\Omega$ while keeping time step fixed. These simulations are performed at two fixed time steps of $\tau=0.008 \mathrm{~s}$ and $\tau=0.016$ s. Results are shown Fig. 2. The DM scheme results appear to be highly sensitive to the rotation rate applied in spite of the fact the solution of the equations are independent of rotation rate. The RIPC scheme with $(q, r)=(3,2)$ shows only slight response to varying rotation rate, while the accuracy of the DM scheme deteriorates significantly as $\Omega$ increases and is less accurate than the RIPC scheme with $(q, r)=(3,2)$. Just for the sake of comparison, we have also plotted the results by RIPC scheme with $(q, r)=(3,1)$.

As the second case, we utilize forcing functions such that the exact solution to (3.1a)(3.1b) depends on $\Omega$ as follows:

$$
\begin{aligned}
& u=\sin (x) \cos (y) \cos (z) e^{\sin (\Omega t)}, \\
& v=-\cos (x) \sin (y) \cos (z) e^{\sin (\Omega t)}, \\
& w=0, \\
& p=\sin (x) \cos (y) \sin (z) e^{\sin (\Omega t)} .
\end{aligned}
$$

The $L_{2}$ error norms are shown in Fig. 3. In this case, the accuracy of both RIPC and DM schemes deteriorates as $\Omega$ increases, and the DM scheme is slightly more accurate than the RIPC scheme with $(q, r)=(3,2)$.

\subsection{Laminar flow simulation}

In this subsection we consider the Taylor-Green Vortex (TGV) flow subjected to solidbody rotation, and report on the performance of the RIPC and MD methods. Here, we solve the Navier-Stokes equations (2.1a)-(2.1b) with the initial velocity field given by:

$$
\begin{aligned}
& u=\sin (x) \cos (y) \cos (z), \\
& v=-\cos (x) \sin (y) \cos (z), \\
& w=0 .
\end{aligned}
$$




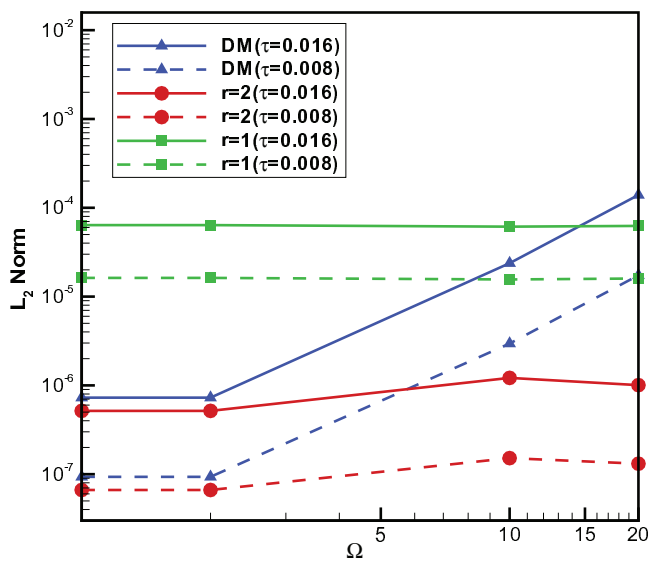

Figure 2: Error Norm variation at different rotation rate $(\Omega)$ and time steps.

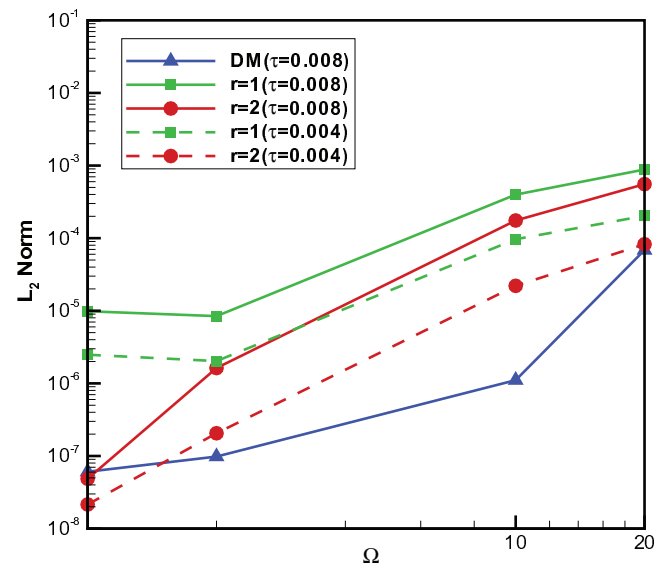

Figure 3: $L_{2}$ Error Norm variation at different rotation rate $(\Omega)$ and time steps.

In what follows, the previously described norms are used to quantify the difference between the two schemes.

\subsubsection{Effect of order of pressure extrapolation}

A rotation rate of $\Omega=20 \mathrm{rad} / \mathrm{s}$ is resolved on a $120^{3}$ grid using the DM scheme and the RIPC scheme with $(q, r)=(2,2)$ and $(q, r)=(2,1)$. Fig. 4 shows velocity magnitude contours with velocity vectors from a simulation using a fixed time step of $\Delta t=4.28 \times 10^{-4}$ $\mathrm{s}$ (obtained based on the CFL constraint). The three results are visually identical.

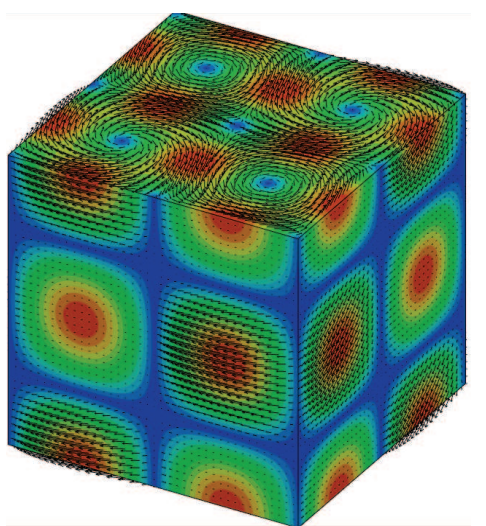

(a) $\operatorname{RIPC}(q, r)=(2,1)$

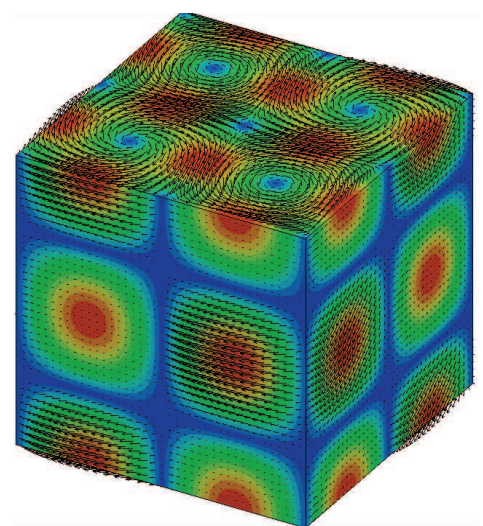

(b) $\operatorname{RIPC}(q, r)=(2,2)$

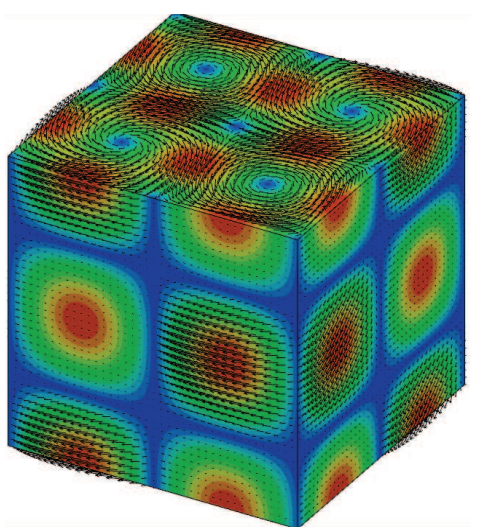

(c) DM Scheme

Figure 4: Velocity magnitude contours using the RIPC and DM schemes.

To better understand the influence of pressure extrapolation order, errors of the firstand second-order pressure extrapolations with the DM method are computed and tabu- 
Table 1: Errors of the RIPC scheme with $(q, r)=(2,1)$.

\begin{tabular}{||c|c|c|c|c||}
\hline$\tau(\mathrm{s})$ & $4.28 \times 10^{-03}$ & $2.14 \times 10^{-03}$ & $4.28 \times 10^{-04}$ & $2.14 \times 10^{-04}$ \\
\hline$L_{2}$ & & & & \\
$\mathrm{u}$ & $3.567 \times 10^{-02}$ & $9.276 \times 10^{-03}$ & $3.666 \times 10^{-04}$ & $9.105 \times 10^{-05}$ \\
$\mathrm{v}$ & $3.567 \times 10^{-02}$ & $9.276 \times 10^{-03}$ & $3.674 \times 10^{-04}$ & $9.122 \times 10^{-05}$ \\
$\mathrm{w}$ & $2.523 \times 10^{-02}$ & $5.970 \times 10^{-03}$ & $2.907 \times 10^{-04}$ & $8.456 \times 10^{-05}$ \\
\hline$H_{1}$ & & & & \\
$\mathrm{u}$ & $7.139 \times 10^{-02}$ & $1.856 \times 10^{-02}$ & $7.337 \times 10^{-04}$ & $1.823 \times 10^{-04}$ \\
$\mathrm{v}$ & $7.139 \times 10^{-02}$ & $1.856 \times 10^{-02}$ & $7.353 \times 10^{-04}$ & $1.826 \times 10^{-04}$ \\
$\mathrm{w}$ & $5.051 \times 10^{-02}$ & $1.195 \times 10^{-02}$ & $5.820 \times 10^{-04}$ & $1.693 \times 10^{-04}$ \\
\hline$L_{\infty}$ & & & & \\
$\mathrm{u}$ & $9.451 \times 10^{-02}$ & $2.487 \times 10^{-02}$ & $9.528 \times 10^{-04}$ & $2.290 \times 10^{-04}$ \\
$\mathrm{v}$ & $9.451 \times 10^{-02}$ & $2.487 \times 10^{-02}$ & $9.567 \times 10^{-04}$ & $2.286 \times 10^{-04}$ \\
$\mathrm{w}$ & $7.131 \times 10^{-02}$ & $1.687 \times 10^{-02}$ & $8.218 \times 10^{-04}$ & $2.391 \times 10^{-04}$ \\
\hline
\end{tabular}

Table 2: Errors of the RIPC scheme with $(q, r)=(2,2)$.

\begin{tabular}{||c|c|c|c|c||}
\hline$\tau(\mathrm{s})$ & $4.28 \times 10^{-03}$ & $2.14 \times 10^{-03}$ & $4.28 \times 10^{-04}$ & $2.14 \times 10^{-04}$ \\
\hline$L_{2}$ & & & & \\
$\mathrm{u}$ & $4.625 \times 10^{-03}$ & $5.623 \times 10^{-04}$ & $3.439 \times 10^{-05}$ & $1.694 \times 10^{-05}$ \\
$\mathrm{v}$ & $4.627 \times 10^{-03}$ & $5.645 \times 10^{-04}$ & $3.163 \times 10^{-05}$ & $1.661 \times 10^{-05}$ \\
$\mathrm{w}$ & $5.605 \times 10^{-03}$ & $7.269 \times 10^{-04}$ & $5.037 \times 10^{-05}$ & $2.560 \times 10^{-05}$ \\
\hline$H_{1}$ & & & & \\
$\mathrm{u}$ & $9.262 \times 10^{-03}$ & $1.127 \times 10^{-03}$ & $6.954 \times 10^{-05}$ & $3.410 \times 10^{-05}$ \\
$\mathrm{v}$ & $9.266 \times 10^{-03}$ & $1.131 \times 10^{-03}$ & $6.409 \times 10^{-05}$ & $3.342 \times 10^{-05}$ \\
$\mathrm{w}$ & $1.121 \times 10^{-02}$ & $1.454 \times 10^{-03}$ & $1.010 \times 10^{-04}$ & $5.146 \times 10^{-05}$ \\
\hline$L_{\infty}$ & & & & \\
$\mathrm{u}$ & $1.055 \times 10^{-02}$ & $1.250 \times 10^{-03}$ & $7.731 \times 10^{-05}$ & $3.505 \times 10^{-05}$ \\
$\mathrm{v}$ & $1.055 \times 10^{-02}$ & $1.252 \times 10^{-03}$ & $7.349 \times 10^{-05}$ & $3.660 \times 10^{-05}$ \\
$\mathrm{w}$ & $1.583 \times 10^{-02}$ & $2.052 \times 10^{-03}$ & $1.429 \times 10^{-04}$ & $7.057 \times 10^{-05}$ \\
\hline
\end{tabular}

lated in Table 1 and Table 2, respectively. The tables also show the effect of time step on the error norms. Note that the results in the last column of Table 2 is not reliable due to the limited resolution used. One observes that the RIPC scheme with $(q, r)=(2,1)$ leads to essentially second-order convergence rate, while the convergence rate of the RIPC scheme with $(q, r)=(2,2)$ seems to be slightly better than second-order. More importantly, the errors with $(q, r)=(2,1)$ is significantly larger than that obtained with $(q, r)=(2,2)$. Therefore, in all simulations below, we shall use $(q, r)=(2,2)$ in the RIPC scheme.

\subsubsection{Effect of rotation rate}

The stability of the scheme over a wide range of rotation rates is investigated here. Three rotation rates, $\Omega=2 \mathrm{rad} / \mathrm{s}, 20 \mathrm{rad} / \mathrm{s}$, and $50 \mathrm{rad} / \mathrm{s}$, are simulated using the RIPC scheme 
Table 3: Errors of RIPC scheme with $(q, r)=(2,2)$ at different rotation rate.

\begin{tabular}{||c|c|c|c||}
\hline$\Omega(\mathrm{rad} / \mathrm{s})$ & 2 & 20 & 50 \\
\hline$L_{2}$ & & & \\
$\mathrm{u}$ & $4.679 \times 10^{-05}$ & $3.439 \times 10^{-05}$ & $1.371 \times 10^{-04}$ \\
$\mathrm{v}$ & $4.676 \times 10^{-05}$ & $3.163 \times 10^{-05}$ & $1.389 \times 10^{-04}$ \\
$\mathrm{w}$ & $4.751 \times 10^{-05}$ & $5.037 \times 10^{-05}$ & $2.105 \times 10^{-04}$ \\
\hline$H_{1}$ & & & \\
$\mathrm{u}$ & $9.843 \times 10^{-05}$ & $6.954 \times 10^{-05}$ & $2.745 \times 10^{-04}$ \\
$\mathrm{v}$ & $9.839 \times 10^{-05}$ & $6.409 \times 10^{-05}$ & $2.783 \times 10^{-04}$ \\
$\mathrm{w}$ & $9.680 \times 10^{-05}$ & $1.010 \times 10^{-04}$ & $4.213 \times 10^{-04}$ \\
\hline$L_{\infty}$ & & & \\
$\mathrm{u}$ & $1.357 \times 10^{-04}$ & $7.731 \times 10^{-05}$ & $2.964 \times 10^{-04}$ \\
$\mathrm{v}$ & $1.326 \times 10^{-04}$ & $7.349 \times 10^{-05}$ & $3.056 \times 10^{-04}$ \\
$\mathrm{w}$ & $1.394 \times 10^{-04}$ & $1.429 \times 10^{-04}$ & $5.960 \times 10^{-04}$ \\
\hline
\end{tabular}

with $(q, r)=(2,2)$ and are compared with the results from the MD scheme. The results are presented in Table 3. All these simulations are performed at a fixed time step of $\tau=4.28 \times 10^{-04} \mathrm{~s}$ using $120^{3}$ grid points. The present scheme is found to be robust at higher rotation rates and the results are in very good agreement with the MD scheme.

\section{Turbulence flow simulation}

In this section, we use the RIPC scheme with $(q, r)=(2,2)$ to simulate rotating turbulent flows using both large eddy simulation (LES) and direct numerical simulation (DNS), and compare the results with the DM scheme.

\subsection{Large eddy simulation}

In LES, the low-pass spatially filtered incompressible Navier-Stokes equations, closed with the Smagorinsky eddy-viscosity subgrid-scale (SGS) turbulence model, are considered. Since the focus of this paper is not to evaluate the suitability of LES subgrid models for rotating turbulence, we have used a the classical Smagorinsky model with a constant model coefficient $=0.16$. Detail of the LES equation and the SGS model can be found in [15]. The numerical integration of these equations is the same as before with the additional SGS stress term treated in the same way as the non-linear convection term.

The velocity field is initialized with the following spectrum (see [4]):

$$
E(k, 0)=A k^{4} \exp \left(-B k^{2}\right),
$$

where $k$ represents the wave number and $A$ and $B$ are constants. The simulation is performed using a kinematic viscosity of $0.005 \mathrm{~m}^{2} / \mathrm{s}$ and rotation rates of $\Omega=5 \mathrm{rad} / \mathrm{s}$ and $50 \mathrm{rad} / \mathrm{s}$. Non-rotating simulations are started with the aforementioned spectrum and 


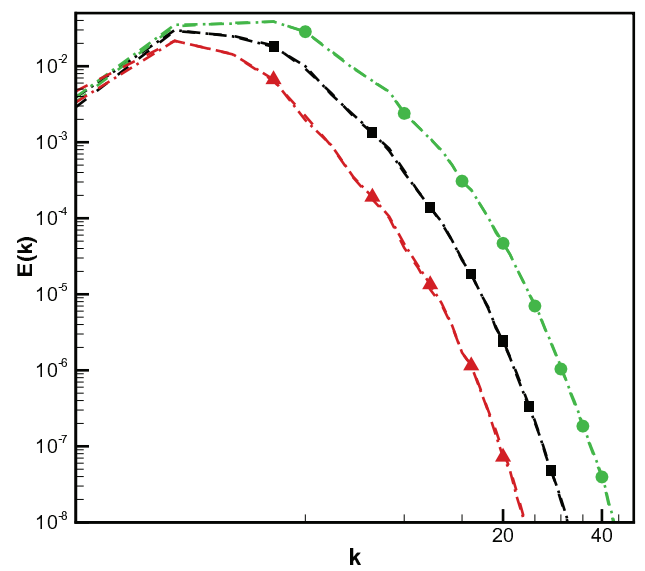

(a) $\Omega=5 \mathrm{rad} / \mathrm{s}$

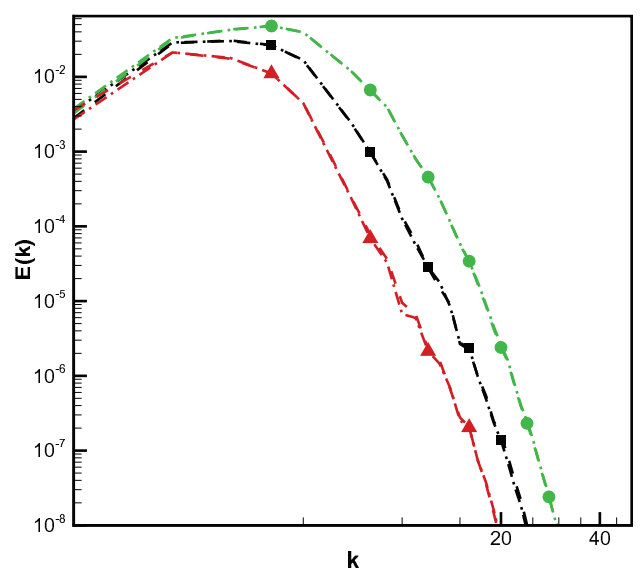

(b) $\Omega=50 \mathrm{rad} / \mathrm{s}$

Figure 5: Energy spectrum obtained for the LES study; dashed line-DM method, dot-dash line-RIPC $[(q, r)=(2,2)]$; Symbols: green circle- $t=2.5 \mathrm{~s}$, black square- $t=5.0 \mathrm{~s}$, red diamond- $\mathrm{t}=7.5 \mathrm{~s}$.

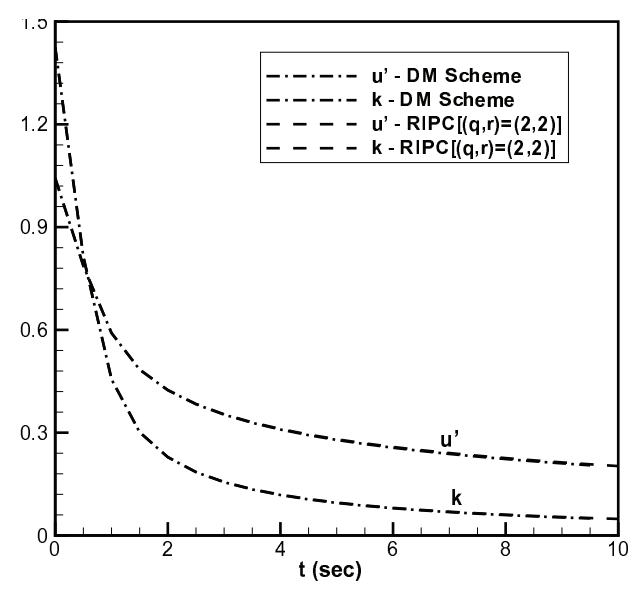

(a) $\Omega=5 \mathrm{rad} / \mathrm{s}$

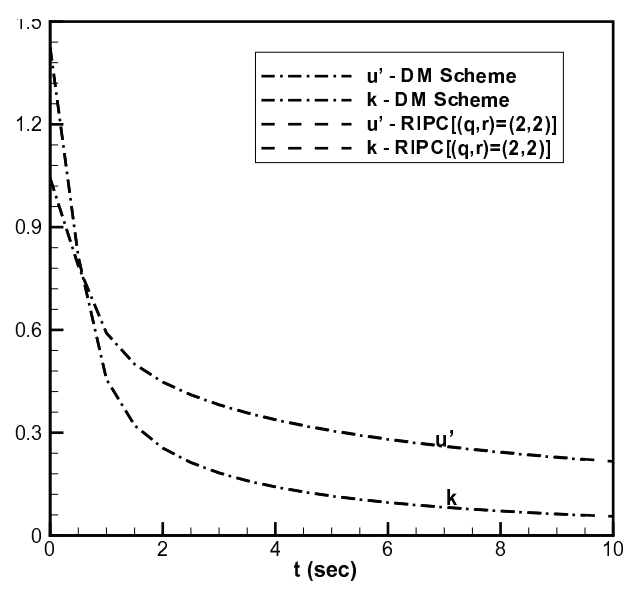

(b) $\Omega=50 \mathrm{rad} / \mathrm{s}$

Figure 6: Rms velocity and the the plot for the LES study.

run up to $1 \mathrm{~s}$, after which solid body rotation is applied to the system. The LES studies are performed using both the RIPC and MD schemes on a $40^{3}$ grid in a $2 \pi$ periodic box. Energy spectra at three different times from the simulations at the two rotation rates are shown in Figs. 5(a) and 5(b). The temporal evolution of the rms velocity and turbulent kinetic energy (tke) from both methods are shown in Figs. 6(a) and 6(b). Very good agreement is observed between the two methods. 
Table 4: Comparison of Schemes for DNS run.

(a) Turbulent Kinetic Energy.

\begin{tabular}{||c|c|c||}
\hline time $(\mathrm{s})$ & DM scheme & RIPC $[(\mathrm{q}, \mathrm{r})=(2,2)]$ \\
\hline 1.0 & 0.6469 & 0.6468 \\
\hline 2.0 & 0.2943 & 0.2945 \\
\hline 3.0 & 0.1941 & 0.1944 \\
\hline
\end{tabular}

(b) Rms Velocity.

\begin{tabular}{||c|c|c||}
\hline time $(\mathrm{s})$ & DM scheme & $\operatorname{RIPC}[(\mathrm{q}, \mathrm{r})=(2,2)]$ \\
\hline 1.0 & 0.6965 & 0.6966 \\
\hline 2.0 & 0.4777 & 0.4780 \\
\hline 3.0 & 0.3923 & 0.3927 \\
\hline
\end{tabular}

\subsection{Direct numerical simulation}

Finally, a short-duration direct numerical simulation (DNS) run of the above case on a $128^{3}$ mesh is carried out for the RIPC and MD schemes. The rotation rate was $\Omega=5$ $\mathrm{rad} / \mathrm{s}$. Turbulent kinetic energy and rms velocity predictions are shown in Table 4, while energy spectra are shown in Fig. 7. Good agreement between both methods is observed. Discrepancies between the DNS and LES results can be attributed to the fact that the Smagorinsky SGS model has difficulties in handling rotating turbulent flows [1].

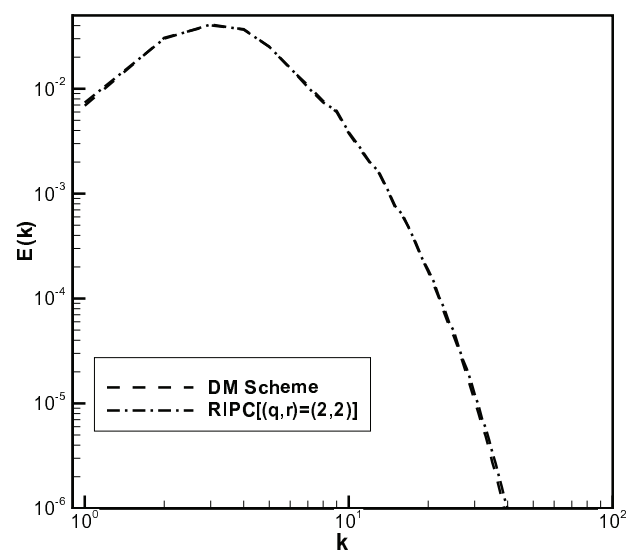

Figure 7: Energy spectrum obtained for the DNS study.

\section{Conclusions}

We introduced in this paper the rotational incremental pressure-correction (RIPC) scheme for rotational Navier-Stokes equations, and proved that the scheme with $(q, r)=(1,1)$ is 
unconditionally stable when applied to the forced Stokes equations. We developed a fast pseudo Fourier-spectral algorithm for the implementation of this scheme in the case of periodic boundary conditions. We implemented this algorithm and applied it to several rotating laminar and turbulent flows.

We made a careful comparison of the RIPC scheme with the diagonalization method (DM) in various situations, including analytical solutions, laminar rotating flows and LES and DNS for turbulent rotating flows. We showed that the results given by the RIPC scheme with the second-order pressure extrapolation are in excellent agreement with the DM method. However, the RIPC scheme is much simpler to implement and computationally more efficient. Moreover, the RIPC scheme is not in anyway limited to implementation in a pseudo-spectral code or periodic boundary conditions, and can be used in complex geometries and with other suitable boundary conditions.

\section{Acknowledgments}

This work is partially supported by NSF grants CBET-0651788 and DMS-0915066.

\section{References}

[1] H. Luo, C. J. Rutland and L. M. Smith, A-priori tests of one-equation LES modeling of rotating turbulence, J. Turbul., 37 (2007).

[2] P. K. Yeung and Ye Zhou, Numerical study of rotating turbulence with external forcing, Phys. Fluids., 10(DOI:10.1063/1.869810), 1998.

[3] L. M. Smith and F. Waleffe, Transfer of energy to two-dimensional large scales in forced rotating three-dimensional turbulence, Phys. Fluids., 11 (1999), 1608-1622.

[4] Y. Morinishi, K. Nakabayashi and S. Q. Ren, A new DNS algorithm for rotating homogeneous decaying turbulence, Int. J. Heat. Fluid. Flow., 22 (2001), 30-38.

[5] A. J. Chorin, Numerical solution of the Navier-Stokes equations, Math. Comput., 22 (1968), $745-762$.

[6] R. Temam, Sur l'approximation de la solution des équations de Navier-Stokes par la méthode des pas fractionnaires II, Arch. Rat. Mech. Anal., 33 (1969), 377-385.

[7] J. L. Guermond, P. Minev and J. Shen, An overview of projection methods for incompressible flows, Comput. Methods. Appl. Mech. Engrg., 195 (2006), 6011-6045.

[8] L. J. P. Timmermans, P. D. Minev and F. N. Van De Vosse, An approximate projection scheme for incompressible flow using spectral elements, Int. J. Numer. Methods. Fluids., 22 (1996), 673-688.

[9] J. L. Guermond and J. Shen, On the error estimates for the rotational pressure-correction projection methods, Math. Comput., 73 (2003), 1719-1737.

[10] J. Shen, Efficient spectral-Galerkin method I, direct solvers for second- and fourth-order equations by using Legendre polynomials, SIAM J. Sci. Comput., 15 (1994), 1489-1505.

[11] J. Shen, Efficient spectral-Galerkin method II, direct solvers for second- and fourth-order equations by using Chebyshev polynomials, SIAM J. Sci. Comput., 16 (1995), 74-87.

[12] R. Temam, Navier-Stokes Equations: Theory and Numerical Analysis, North-Holland, Amsterdam, 1984. 
[13] F. Chen and J. Shen, Efficient spectral methods for solving systems of coupled elliptic equations, preprint, 2010.

[14] R. S. Rogallo, Numerical experiments in homogeneous turbulence, NASA TM., 81315, 1981.

[15] A. J. Chandy and S. H. Frankel, Regularization-based sub-grid scale (SGS) models for large eddy simulations (LES) of high-Re decaying isotropic turbulence, J. Turbul., 10 (2009).

[16] H. Lu, One Equation LES Modeling of Rotating Turbulence, Ph.D Thesis, University of Wisconsin Madison, 2007. 